\title{
Status and results of the OPERA experiment
}

\author{
Valeri Tioukov* \\ INFN Napoli \\ E-mail: valeri@na.infn.it
}

The OPERA neutrino detector built in the underground Gran Sasso Laboratory is designed to detect $v_{\mu} \rightarrow v_{\tau}$ oscillations in direct appearance mode. The $v_{\tau}$ signature detection is based on the $\tau$ short decay topology in the vertex zone. The OPERA hybrid apparatus consists of an emulsion/lead target complemented by electronic detectors. It is placed in the long-baseline CERN to Gran Sasso neutrino beam (CNGS) $730 \mathrm{~km}$ away from the source. OPERA is taking data since 2008. A first $v_{\tau}$ interaction candidate was already published in 2010. In this report, the status of the OPERA experiment and statistical significance of the first candidate observed so far are presented.

The XXth International Workshop High Energy Physics and Quantum Field Theory September 24 - October 1, 2011

Sochi, Russia

${ }^{*}$ Speaker. 


\section{Introduction}

OPERA [1] is a hybrid experiment based on electronic detectors and nuclear emulsions. It is exposed to the long-baseline CNGS beam [2] from CERN to the Gran Sasso underground laboratory (LNGS) $730 \mathrm{~km}$ away from the neutrino source. The main purpose of the experiment is the observation of $v_{\mu} \rightarrow v_{\tau}$ oscillations in the direct appearance mode. The $v_{\tau}$ are identified through the measurement of the $\tau$ leptons produced in their Charged Current (CC) interactions. The neutrino runs started in 2008 and a first $\tau$ candidate has recently been observed [3]. The beam is mainly composed of $v_{\mu}$ interactions due to the $\bar{v}_{\mu}, v_{e}$ and $\bar{v}_{e}$ contamination amount to $2.1 \%, 0.80 \%$ and $0.07 \%$ of the $v_{\mu} \mathrm{CC}$ event rate.

\section{The OPERA hybrid detector structure}

The challenge of the OPERA experiment is to achieve the very high spatial accuracy required for the detection of $\tau$ leptons (whose decay length is of the order of $1 \mathrm{~mm}$ in this experiment) inside a large-mass active target. The hybrid detector [5] is composed of two identical Super Modules (SM), each consisting of an instrumented target section of a mass of about 625 tons followed by a magnetic muon spectrometer (Fig. 1). A target section is a succession of walls filled with elements called bricks, interleaved with planes of scintillator strips, the Target Tracker (TT). The TT allows the initial localization of neutrino interaction by providing also its time stamp. A brick is an Emulsion Cloud Chamber (ECC) module consisting of 56 lead plates of $1 \mathrm{~mm}$ interleaved with 57 nuclear emulsion films. It weights $8.3 \mathrm{~kg}$ and its thickness corresponds to 10 radiation lengths along the beam direction. Tightly packed removable doublets of emulsion films called Changeable Sheets (CS) are glued to the downstream face of each brick. They serve as interfaces between the TT planes and the bricks to facilitate the location of neutrino interactions. Complex brick handling ancillary facilities are used to bring emulsion films from the target up to the automatic scanning microscopes [6]. An emulsion film is made of two layers of nuclear emulsion gel 44 $\mu m$ thick deposited on each side of a $205 \mu \mathrm{m}$ thick plastic base of $12.5 \times 9.9 \mathrm{~cm}^{2}$ [7] . Emulsion records charged particles as 3D tracks with sub-micron resolution. ECC technique is adequate to recognize $\tau$ decay topologies as proven by the DONuT experiment [10]. Completely automated emulsion scanning systems based on the computer-driven optical microscopes used to extract from the emulsions tracks and vertex information(Fig. 2).

\section{Location of neutrino interactions}

The experiment is currently in the phase of its fourth year of data taking and of data analysis. The CNGS neutrino beam status shown in Table 1. The total number of protons on target was $12.65 \times 10^{19}$ as of 20 Aug. 2011. The results presented in this report are based on the decay search analysis of 2738 events from 2008 and 2009 samples. In order to analyse the primary vertex a volume scan is performed over a $1 \mathrm{~cm}^{2}$ area in at least 2 films upstream and 6 films downstream of the vertex lead plate. When a secondary vertex is found the kinematic analysis of the whole event is performed. This analysis makes use of the angles measured in the emulsion films, the momenta determined by multiple Coulomb scattering as measured in the brick, the momenta measured by 


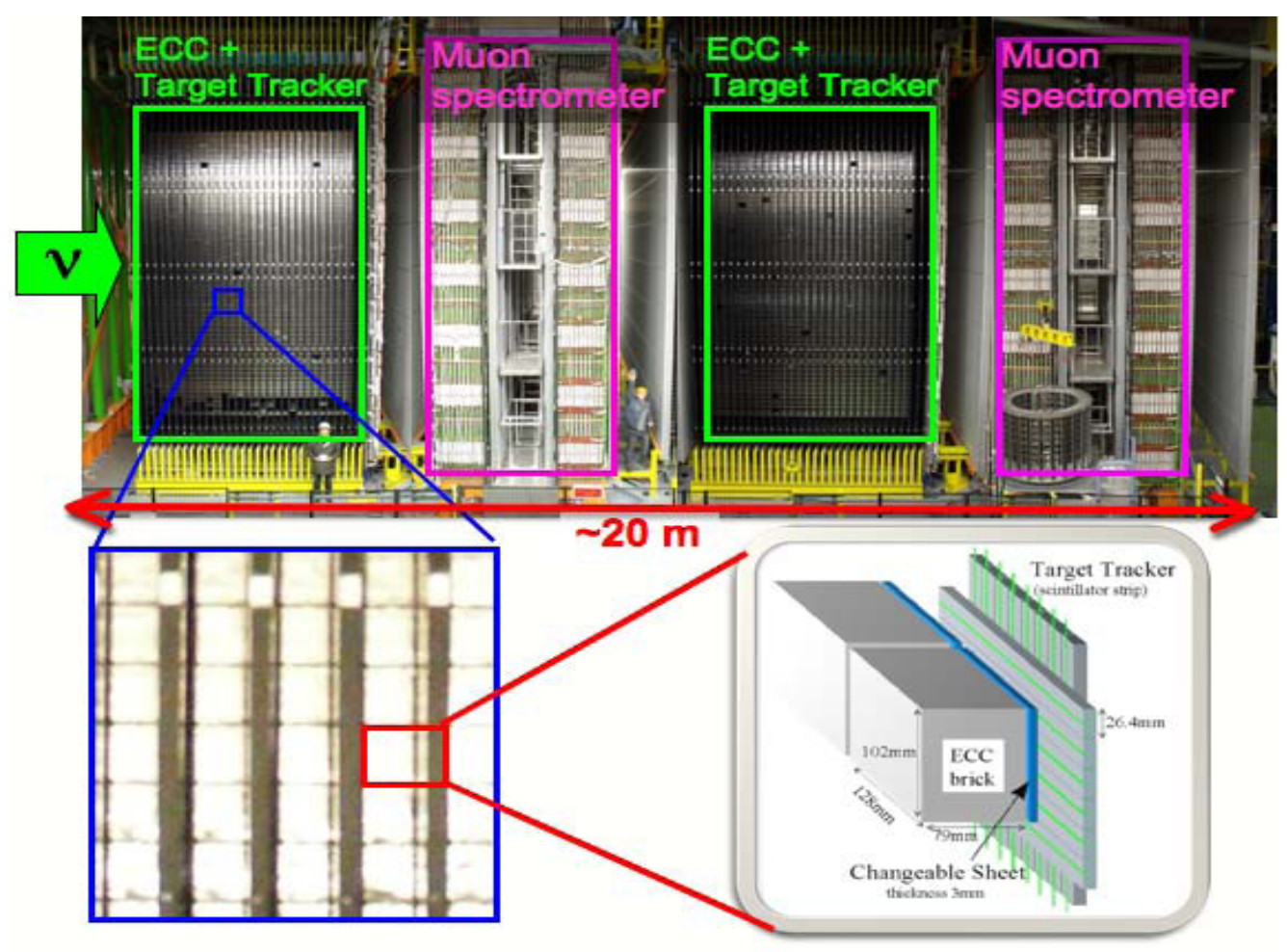

Figure 1: Sketch of the OPERA detector with walls consisting of ECC bricks

the magnetic spectrometers, and the total energy deposited in the instrumented target acting as a calorimeter [9-11]. By applying this procedure, the first $v_{\tau}$ candidate event was observed in 2010, as reported in detail in [9].

\begin{tabular}{|c|c|c|c|}
\hline Year & Beam days & Protons on target & Events in the bricks \\
\hline 2008 & 123 & $1.78 \times 10^{19}$ & 1698 \\
2009 & 155 & $3.52 \times 10^{19}$ & 1698 \\
2010 & 187 & $4.04 \times 10^{19}$ & 1698 \\
2011 & Ongoing & $3.31 \times 10^{19}$ & 3304 \\
\hline
\end{tabular}

Table 1: Status of CNGS neutrino beam as of 20 Aug. 2011

\section{Signal and background}

Charged charmed particles own lifetimes similar to that of the $\tau$ lepton and share analogous decay topologies. The finding efficiency of the decay vertices is therefore also similar for both types of particles. Comparing the observed charm event sample in size, decay topologies and kinematics with expectations from simulations constitutes a direct way to verify prompt-decay selection criteria and their corresponding efficiencies as well as backgrounds evaluations. Recently published cross-sections by the CHORUS experiment [13] have been used in the simulation. The 

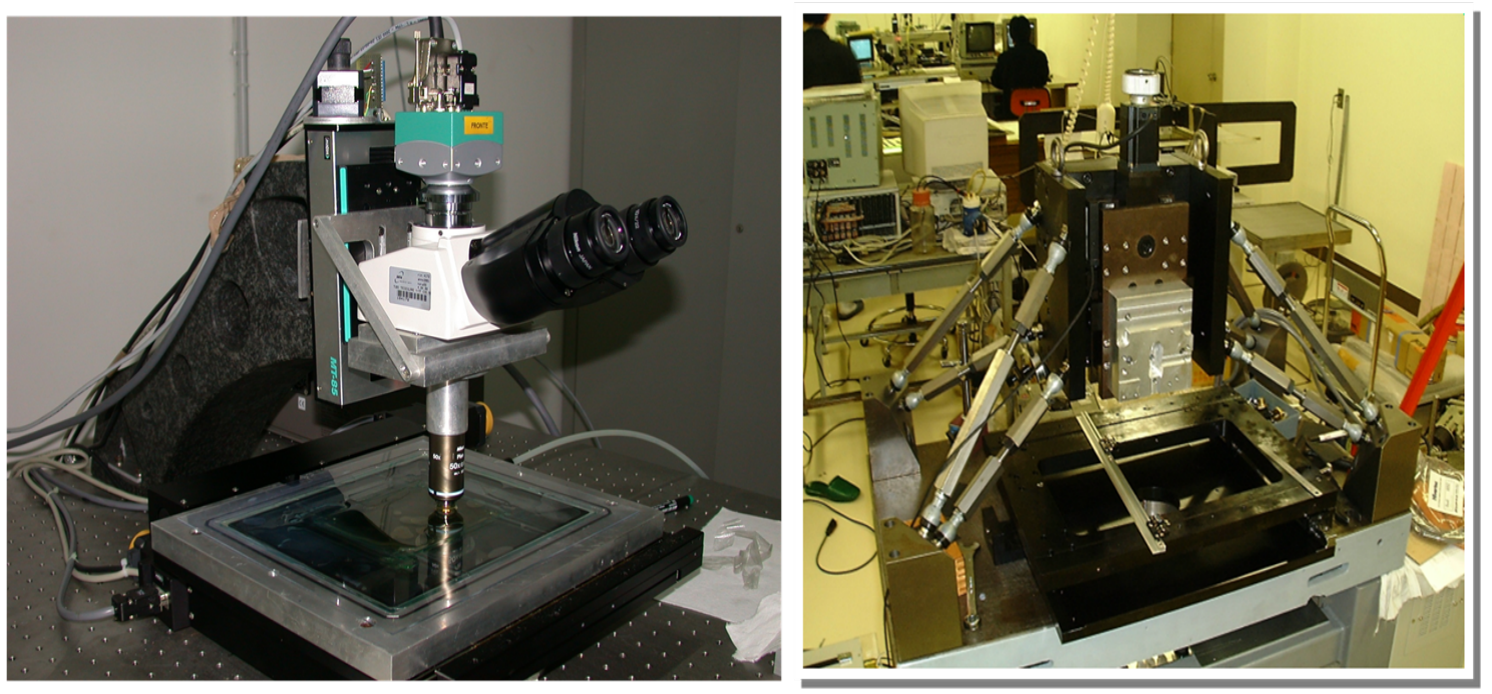

Figure 2: European scanning system (left) and Japanese SUTS (right)

\begin{tabular}{|l|c|c|}
\hline Decay channel & $22.5 \times 10^{19}$ p.o.t. & Analysed sample \\
\hline$\tau \rightarrow \mu$ & 1.79 & 0.39 \\
$\tau \rightarrow e$ & 2.89 & 0.63 \\
$\tau \rightarrow h$ & 2.25 & 0.49 \\
$\tau \rightarrow 3 h$ & 0.71 & 0.15 \\
\hline Total & 7.63 & 1.65 \\
\hline
\end{tabular}

Table 2: Number of signal events expected in OPERA for $\Delta m^{2}=2.5 \times 10^{-3} \mathrm{eV}^{2}$

expected numbers of events in the various $\tau$ decay channels for the nominal number of $22.5 \times 10^{19}$ p.o.t. and for the fraction of the 2008 and 2009 runs analysed so far are shown in Table 2. Full mixing and $\Delta m_{23}^{2}=2.5 \times 10^{-3} \mathrm{eV}^{2}$ are assumed. The total number of signal events expected to be eventually detected has decreased from 10 as quoted in the experiment proposal [11] to 8. This reduction is essentially due to the lower efficiency in location of the interaction vertex resulting from a more reliable knowledge of the detector and of the analysis procedures. The main source of background to all $\tau$ decay channels is constituted by charged charmed particles that decay into similar channels and are produced in $v_{\mu}$ CC interactions where the primary muon is not identified. The second main source of background in the $\tau \rightarrow h$ decay channel comes from oneprong inelastic interactions of primary hadrons produced in $\mathrm{NC}$ interactions, or in CC interactions where the primary lepton is not identified, and in which no nuclear fragments can be associated with the secondary interaction. This has been evaluated with Monte Carlo Simulation and crosschecked with measurements. The expected background in the muon decay channel caused by large angle muon scattering has been evaluated in [11]. All background sources are summarized in Table 3. Systematic errors of $25 \%$ on charm background and of $50 \%$ on hadron and muon backgrounds are assumed. Errors arising from the same source are combined linearly, otherwise in quadrature. 


\begin{tabular}{|l|c|c|c|c|c|c|c|c|}
\hline \multirow{3}{*}{ Decay channel } & \multicolumn{7}{|c|}{ Number of background events expected for } \\
\cline { 2 - 9 } & \multicolumn{9}{|c|}{$22.5 \times 10^{19}$ p.o.t. } & \multicolumn{5}{c|}{ Analysed sample } \\
\cline { 2 - 9 } & Charm & Hadron & Muon & Total & Charm & Hadron & Muon & Total \\
\hline$\tau \rightarrow \mu$ & 0.025 & 0.00 & 0.07 & $0.09 \pm 0.04$ & 0.00 & 0.00 & 0.02 & $0.02 \pm 0.01$ \\
$\tau \rightarrow e$ & 0.22 & 0.00 & 0.00 & $0.22 \pm 0.05$ & 0.05 & 0.00 & 0.00 & $0.05 \pm 0.01$ \\
$\tau \rightarrow h$ & 0.14 & 0.11 & 0.00 & $0.24 \pm 0.06$ & 0.03 & 0.02 & 0.00 & $0.05 \pm 0.01$ \\
$\tau \rightarrow 3 h$ & 0.18 & 0.00 & 0.00 & $0.18 \pm 0.04$ & 0.04 & 0.00 & 0.00 & $0.04 \pm 0.01$ \\
\hline Total & 0.55 & 0.11 & 0.07 & $0.73 \pm 0.15$ & 0.12 & 0.02 & 0.02 & $0.16 \pm 0.03$ \\
\hline
\end{tabular}

Table 3: Expected numbers of observed background events from different sources for the nominal number of $22.5 \times 10^{19}$ p.o.t. and for the analysed sample of the data accumulated in the 2008 and 2009 runs. The quoted errors are systematic ones.

\section{Neutrino velocity measurement}

Long baseline $(730 \mathrm{~km})$ experiment is well suited for the precise neutrino velocity measurement. Using the modern GPS and geodesic methods the distance between the proton beam target at CERN and the OPERA detector was measured with the accuracy of $20 \mathrm{~cm}$. Using GPS "common view" technique the synchronization link of $1 \mathrm{~ns}$ accuracy between CERN and OPERA DAQ systems was established. Using the statistics of about 16000 events and considering the presence of the other sources of errors in the beam line, detector and the DAQ systems on the both sides the overall accuracy of $10 \mathrm{~ns}$ can be achieved. In September 2011 the preliminary result of $60 \mathrm{~ns}$ neutrino anticipation in respect to the speed of light was reported. Presently (Jan-2012) performed cross check and remeasuring of all elements of the synchronization and acquisition chain has identified two issues that could significantly affect the reported result. Collaboration is looking forward to performing a new measurement of the neutrino velocity as soon as a new bunched beam will be available in 2012 .

\section{Conclusion}

The OPERA experiment has completed the study of $92 \%$ of data accumulated during the first two years of operation in the CNGS beam (2008-2009) with the aim of the first detection of neutrino oscillations in direct appearance mode. The observation so far of a single candidate event is compatible with the expectation of 1.65 signal events. The significance of the observation of one decay in the $\tau \rightarrow h$ channel is $95 \%$. Our expectation is to collect by the end of 2012 a total statistics (2008-2012) as close as possible to the goal of the experiment: of $22.5 \times 10^{19}$ p.o.t The analysis of the large event samples collected in the 2010 and 2011 CNGS runs and corresponding to $6.90 \times 10^{19}$ p.o.t. at the moment of writing is in progress. The main physics goal of the experiment remains the statistically significant observation of direct appearance $v_{\mu} \rightarrow v_{\tau}$ flavor transition, as well as of the study of the sub-leading $v_{\mu} \rightarrow v_{e}$ oscillation. 


\section{References}

[1] OPERA collaboration, Guler $\mathrm{M}$ et al. An appearance experiment to search for oscillations in the CNGS beam: experimental proposal CERN-SPSC-2000-028

[2] Elsener K, The CERN neutrino beam to Gran Sasso (conceptual technical design) CERN 98-02 INFN/AE-98/05 CNGS project: http://proj-cngs.web.cern.ch/proj-cngs/

[3] OPERA collaboration, Agafonova N et al. 2010 Phys. Lett. B 691138

[4] OPERA Collaboration, Acquafredda R et al. 2009 JINST 404018

[5] Morishima K and Nakano T 2010 JINST 504011

[6] Armenise N et al. 2005 Nucl. Instrum. Meth. A 551261

[7] De Serio M et al. 2005 Nucl. Instrum. Meth. A 554247

[8] Arrabito L et al. 2006 Nucl. Instrum. Meth. A 568578

[9] Nakamura T et al., 2006 Nucl. Instrum. Meth. A 55680

[10] DONuT Collaboration, Kodama K et al. 2011 Phys. Lett. B 504218

[11] OPERA Collaboration, Agafanova N et al. 2011 New J. Phys. 13053051

[12] OPERA Collaboration, Agafanova N et al. New J. Phys. 14 (2012) 013026, arXiv:1106.6211.

[13] CHORUS Collaboration, Kayis-Topaksu A et al. 2011 New J. Phys. 13093002

[14] OPERA Collaboration, Guler M et al. CERN-SPSC-2000-028, LNGS P25/2000 